\title{
DBS Artifact Removal Through Template Subtraction (March 2019)
}

\author{
Marco Emilio Vazquez, Enrico Opri, Brandon Parks, Gunduz Aysegul \\ University of Florida
}

Faculty mentor: Gunduz Aysegul, Department of Biomedical Engineering

\begin{abstract}
Deep Brain Stimulation (DBS) typically results in the formation of large-signal artifacts in electrophysiological recordings in the surrounding regions of the stimulated area. These additional artifacts can prove problematic, as it makes the study of physiological responses in Local Field Potentials (LFPs), and consequently, Event Related Potentials (ERPs) quite challenging. Research has been conducted in an attempt to attenuate the effects of these large artifacts in various ways, most commonly through blind suppression, function fitting, template subtraction, and adaptive filters. However, many of these methods have proven to only be useful within the context of surface recordings (EEGs) and not for LFPs. In our research, we utilized template subtraction and extended it to the context of LFPs, in an attempt to uncover more effectively the underlying physiological responses to DBS.

Keywords: DBS Artifact Removal, Closed-loop DBS, Local Field Potentials (LFPs), Event Related Potentials (ERPs), Template Subtraction
\end{abstract}

\section{Introduction}

Deep Brain Stimulation (DBS) is an effective and well-known technique in neurological scientific communities for treating movement disorders, such as essential tremor and Parkinson's Disease (PD) (Hashimoto, 2002). The process of DBS involves the implantation of electrode arrays (typically with four contacts) deep into the appropriate brain regions (e.g. subthalamic nucleus) where electrical stimulation is seen to provide symptom suppression. In the past, a technique called 'blanking' was employed, where constant electrical stimulation was used in an attempt to overwhelm a small region. While blanking did prove to be effective in removing any visible disorders, it was not an effective method of providing stimulation, and it did not allow for any signal analysis of the neural networks under stimulation. A better approach is currently being developed in our lab and at others, where the implantable device detects the symptoms of the disorder from brain signals and then sends electrical pulses for as long as the movement disorder is detected. This process is known as closed-loop DBS. However, this therapy is at its infancy and signal analysis of the area under observation is still quite challenging due to stimulation 
artifacts. The device's electrical stimulation causes a stimulation artifact as it passes through the electrode-tissue interface and is sensed by the recording electrodes during the periods in which the device is active. Because stimulus artifacts are often several orders of magnitude larger than the recorded electrophysiological signal of interest (Culaclii, et al., 2018), they can potentially saturate the recording amplifier and obscure the underlying physiological responses. The saturation complicates both real-time and in-vitro analysis of Local Field Potentials (LFPs) and Event Related Potentials (ERPs), both of which are an integral part of understanding the complex neurobiological effects caused by neuromodulation.

Various techniques have been employed in the past to remove this artifact and prevent saturation. These techniques consist of hardware implementation, software implementation, or a combination of both to eliminate the artifact (Culaclii, et al., 2018). Preventing saturation is, however, limited to hardware implementation as saturation naturally occurs at the sampling stage. Within our context, we only focus on artifact removal techniques and assume that saturation is dealt with before processing in the hardware, which is handled by the recording devices. The artifact removal techniques are then further subdivided into 'blind' suppression, form-fitting removals, and template subtraction.

'Blind' suppression artifact removal techniques, such as notch filters (Jech, et al., 2006) and non-linear Hampel filters (Pearson, 2002), are referred to as 'blind' because they do not adapt to the specific artifact and instead uniformly attempt to remove them. Within this category, the notch filter has seen the most success in suppressing the artifact's frequency and its harmonics without phase distortion. Even then, the notch filter is still susceptible to generating distortions from filter overshoots created by a signal's steep gradient.

At the other end of the spectrum lies form-fitting removal techniques, where an artifact is fitted and then subtracted. A notably successful approach in this category was proposed by Harding (1996), who fitted and subtracted a combination of predefined templates in the context of single-unit recordings. However, a potential pitfall in this subcategory of techniques is their rigidity. These techniques rely on the assumption that the artifact is of constant amplitude, length, and shape, which is not the case for many stimulation artifacts, including our own, and so do not apply to diverse scenarios. In addition, Harding's algorithm produces substantial residual artifact in the range of LFP signals, which is unacceptable for LFP analysis (Sun, 2016). 
Our proposed method lies within the third category, template subtraction. An algorithm proposed by Hashimoto et al. (2002) illustrates this technique. A representative spike artifact is first created by averaging a large number of spikes triggered by the spikes' onset. The template is then subtracted from each artifact spike, thus removing the artifact. In his experiment, this technique was applied as part of the analysis of neural spike activity sampled at a rate of $50 \mathrm{kHz}$. The higher rate can pose an issue for LFP recordings, which are typically sampled at a much lower rate. Additionally, this technique does not consider each artifact's shape and amplitude, which may differ from one artifact to another.

Both the lower frequency sampling and the difference in individual artifacts were addressed by Limin Sun et al. (2016). Lower frequency sampling was resolved through first upsampling the recorded signal through sinc interpolation, applying 'Moving Average Subtraction' (MAS) and then downsampling the signal back down to its original sampling frequency. The MAS algorithm naturally accounts for and assumes possible differences among distant spike artifacts because it calculates the template and subtracts it on a 'window' basis. In other words, the algorithm calculates the template of a small section based on a group of spike artifacts and subtracts within that group, then moves onto another section and repeats the process.

However, even with its effectiveness in creating a successful and accurate template for removing most artifacts, the MAS algorithm does not take into consideration arbitrary stimulation time and amplitude changes in artifacts (see Fig. 1). Also, the MAS algorithm is computationally expensive and time-consuming.

Recently, variable amplitude stimulation has been tested to avoid patient discomfort and pain from sudden perceptible changes within the patient's brain. By slowly increasing the amplitude of the voltage of stimulation, the patient can get used to the increasing peak voltage of the stimulation. As clinically helpful as this feature may be, it does pose a problem for algorithms not adapted to removing variable amplitude artifacts such as the MAS algorithm.

Our proposed algorithm is simpler than the MAS algorithm and takes less time to compute. While its current version does not account for possible differences among distant peaks, it can be applied to the amplitude changing artifacts as described above. The template subtraction subcategory was chosen because it fits an artifact's features while still maintaining some flexibility in adapting to a diverse set of artifacts. 


\section{Materials and Methods}

\section{DBS artifact}

When the stimulation is turned on, a very large and visible sinusoid centered around $135 \mathrm{~Hz}$ can be observed in many but not all channels with an initially growing amplitude that stabilizes and remains for as long as the stimulation occurs. The turning off of stimulation is also very notable as the signal reverts to showing physiological recordings (Fig. 1). The signal is preprocessed using high and low pass 2nd order Butterworth filters at 1 and $200 \mathrm{~Hz}$, respectively. They are then passed through notch filters that remove $60 \mathrm{~Hz}$ and its subsequent harmonics. All signals are normalized to the baseline signal.
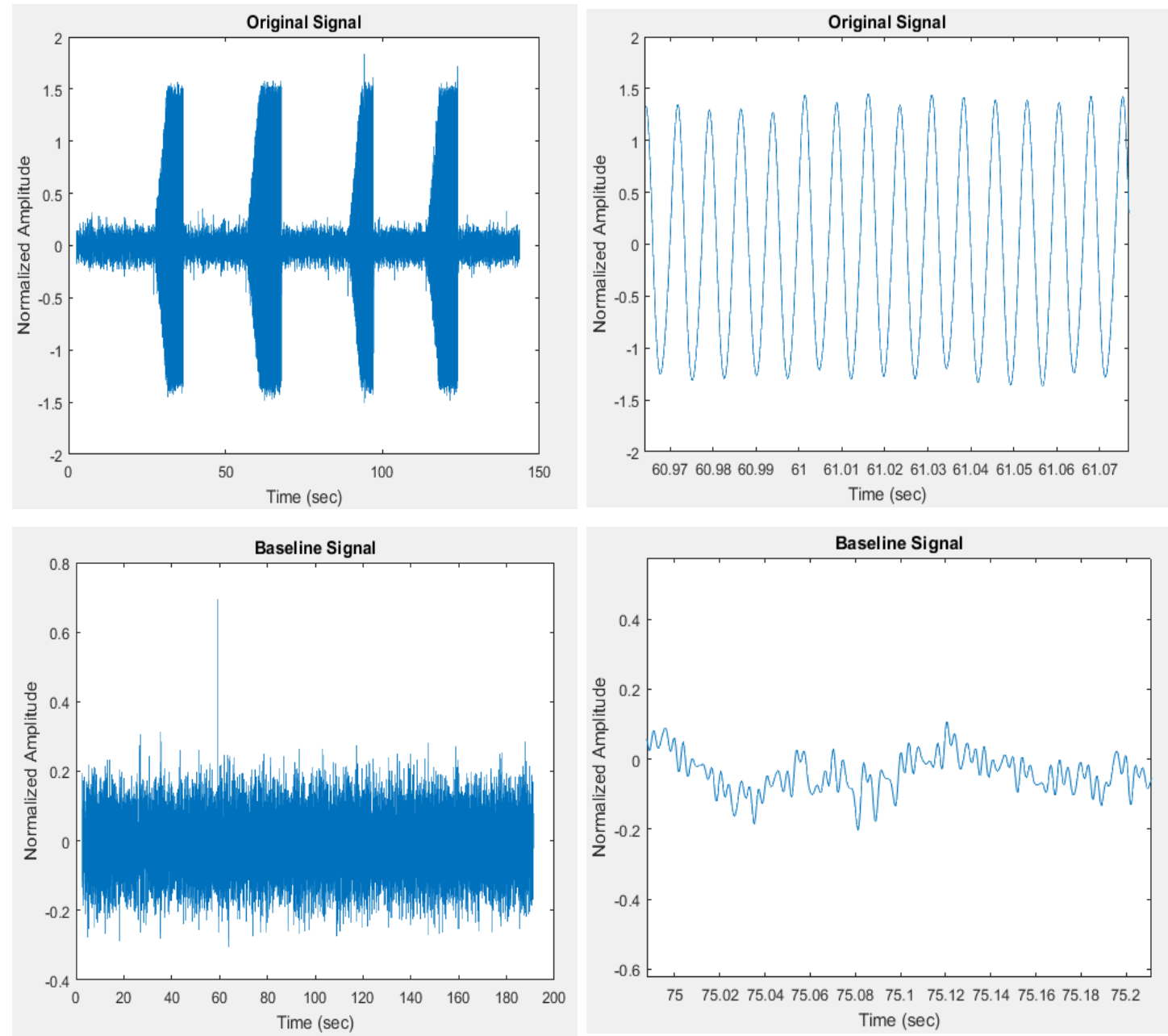

Figure 1. Original Signal (Above) in comparison to the Baseline (Below). The artifact begins by ramping up to a certain amplitude, then stops completely when stimulation is turned off for some time. 


\section{Template Subtraction Algorithm}

The algorithm is divided into four different sections, as follows:

1. Finding Peaks

2. Determining Template Size

3. Creating the Template

4. Subtracting the Template

Finding peaks. We begin by determining at what points the artifact affects the signal by creating a threshold value that is triggered by the arrival of the artifact's distortion. The threshold values are calculated as follows:

Above Threshold $=$ median $(\operatorname{artfree})+\operatorname{range}(\operatorname{artsig}-\operatorname{artfree}) / 16$

Below Threshold $=$ median $(\operatorname{artfree})-\operatorname{range}(\operatorname{artsig}-\operatorname{artfree}) / 16$

where artfree is the 'artifact free' signal; a channel in which the artifact is filtered out using a lowpass filter targeting the frequency of the artifact signal, and artsig is the 'artifact enhanced' signal of the original signal, with its artifact enhanced through a bandpass centered around the target frequency of the artifact. The use of the median as opposed to the mean ensures that the middle point of the signal is chosen, and extreme outliers, which are common when the recording device is affected by external events such as moving the device, do not negatively impact the threshold calculation. The range represents the difference in the artifact enhanced signal and the actual signal that makes the artifact discernible from the physiological signal. The thresholds are triggered by the artifact enhanced signal, artsig, as opposed to the original signal because the original signal may have erroneous triggers from other sources that do not correspond to the artifact.

Determining template size. The template size is determined by finding the most common distances among consecutive peaks appropriate for creating a template that will not overlap nor underlap when subtracting from the original signal. This distance is then used to determine the length of the template that will be created.

Creating the template. Using the distance determined in the previous step, the template is created by collecting templates centered at each local maximum between (local maximum distance/2) and (local maximum + distance/2). The templates are then averaged to create a representative template of the whole set. 
Subtracting the template. Once the template is created, the process of removing the artifact begins. Centered around each local maximum, the template representing the set is first scaled to the amplitude of the area surrounding the local maximum and then subtracted from the original signal at each local maximum.
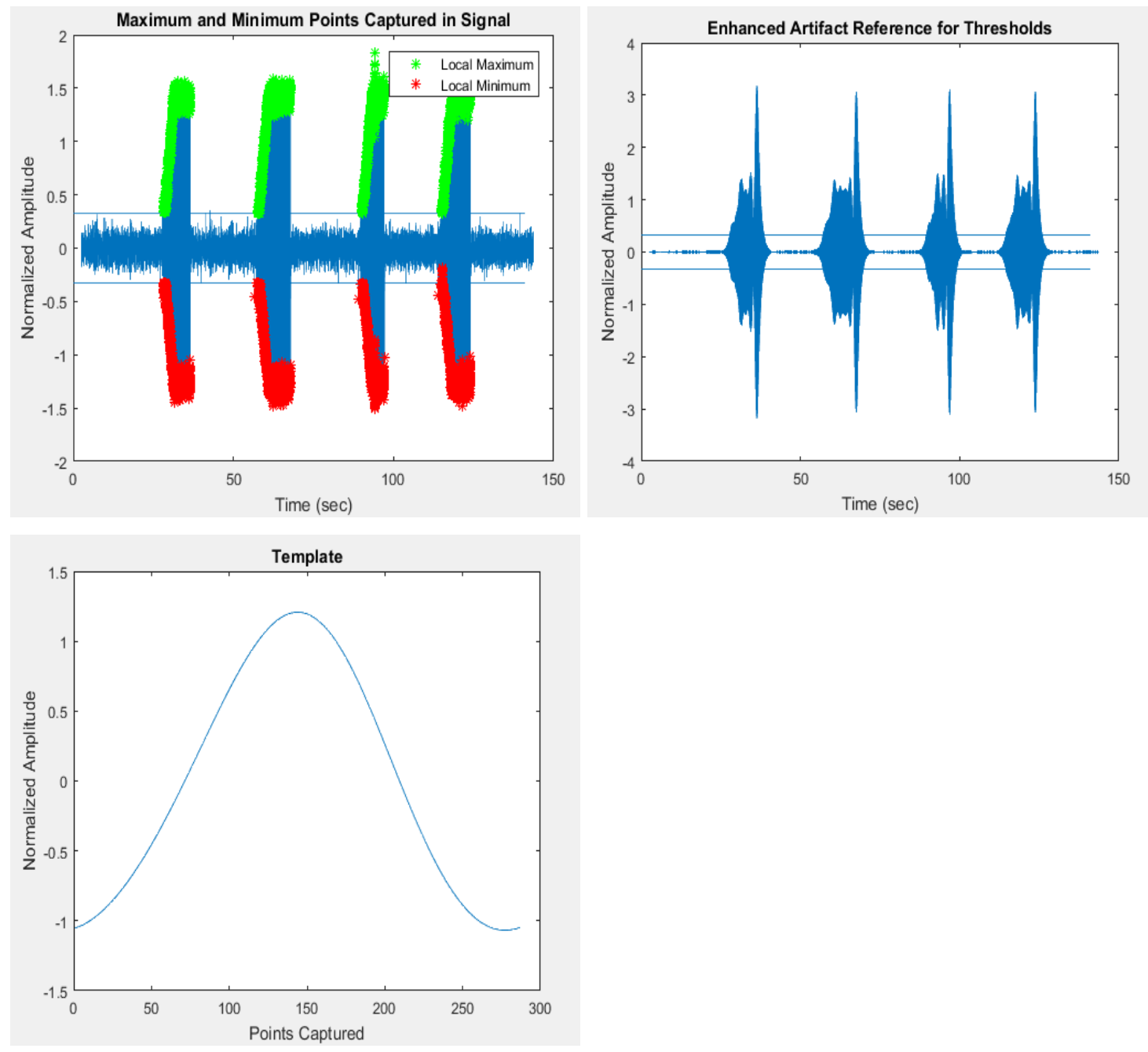

Figure 2. Maximum and minimum points captured above thresholds (Left Above). Enhanced Artifact Reference for Thresholds (artsig) (Right Above). Created Template from average of templates (Left Below).

\section{Results}

The algorithm calculated the results within seconds and demonstrated the power differences in the signals from a spectrogram. A comparison of the original signal and the resulting signal shows the decrease in power centered around $135 \mathrm{~Hz}$ of the artifact that previously had overshadowed the entire signal, and now reveals physiological signals. This test was done across 
all channels and shows the same results in all of them regardless of any amplitude differences in the artifact.

Unfortunately, it is also apparent that the artifact was not entirely removed within the spectrogram, as there are visible traces of the artifact in the resulting signal spectrogram centered around $135 \mathrm{~Hz}$ (Fig. 3). In addition, there are smaller and significantly less perceptible parts of the artifact that were not detected and not filtered by the algorithm as seen in the region after the artifact's onset. These differences can be seen across all channels.
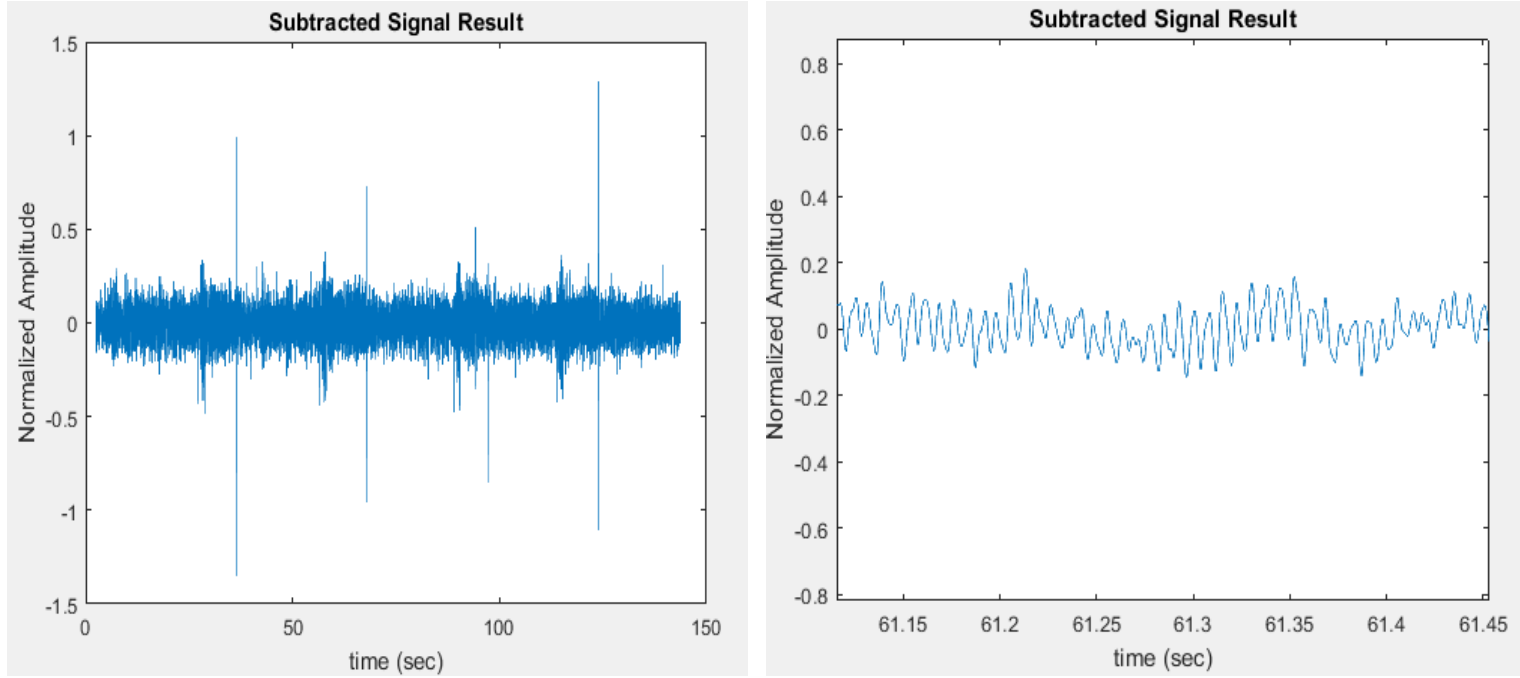

Figure 3. Resulting Signal after template subtraction. The artifact presence has decreased significantly but is faintly visible.
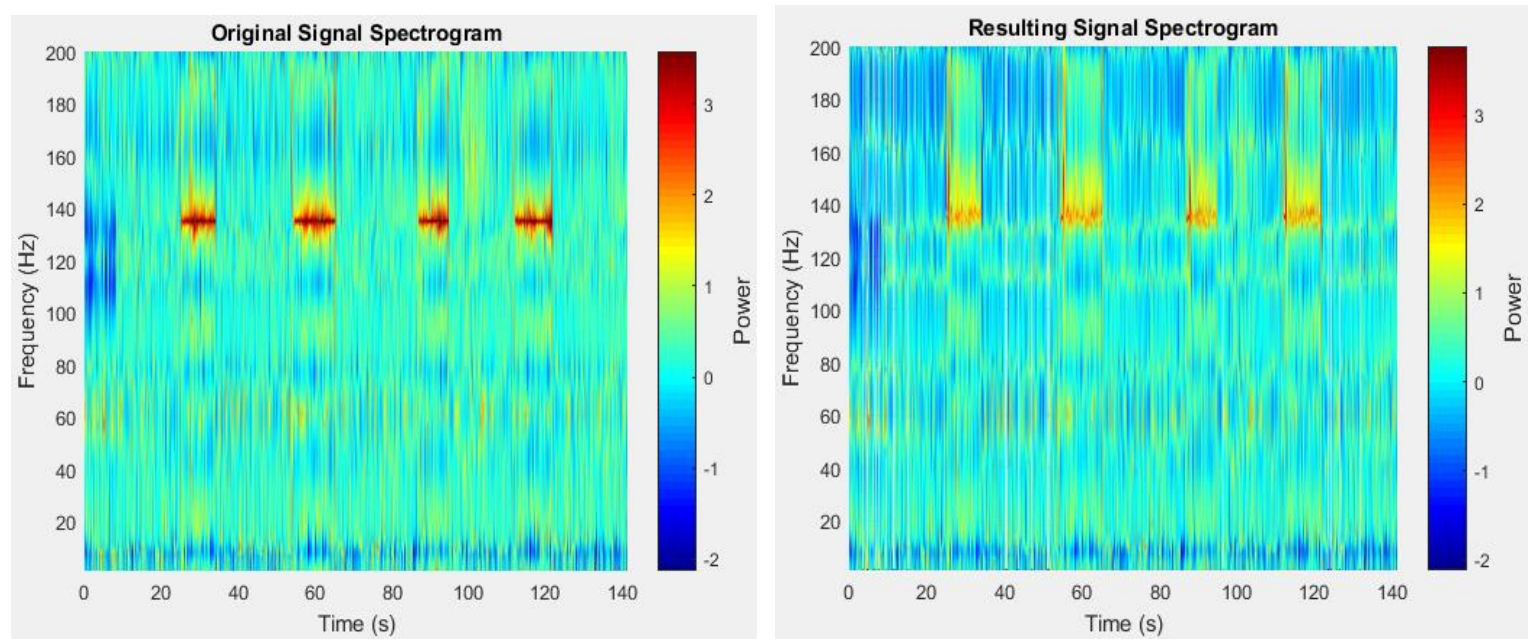

Figure 4. Side by side spectrogram comparison of original signal (Left) to resulting signal (Right). The resulting signal has a clear decrease in artifact power without any visible distortion, but is not entirely removed as seen by small traces centered around $135 \mathrm{~Hz}$. 


\section{Conclusion}

While the algorithm was capable of removing most of the artifact and revealing the underlying physiological signals, it was not able to entirely remove the artifact. The remaining traces of the artifact is possibly due to the template not entirely fitting the artifact due to individual differences among distant peaks of the artifact. These differences seem akin to those of spike type artifacts. Nevertheless, as a further step, the algorithm can be improved upon to create a moving average template that can better fit those individual differences of the peaks at the cost of computational power.

The smaller areas not detected by the algorithm would pose a more challenging problem, as they are almost inseparable from the physiological signals if it were not for the enhanced artifact version of the signal (artsig). It may prove challenging to remove these areas, as reducing the thresholds to detect them might only skew the template creation by erroneously fitting physiological signals. A possibility in removing these might be to use the already created templates in the larger sections to subtract them off, but this would not account for the problem of the individual difference. Better methods of detecting this smaller amplitude artifact section have yet to be discovered.

In the future steps, we may want to consider improvements to the current template subtraction algorithm through a moving average method as well as other techniques such as parametric fitting. The moving average method can be integrated to account for the amplitude changes in the artifact, and empirical tests can be done to determine both the optimal window of templates and artifact size to remove the artifact best. Parametric fitting would fall under the subcategory of form-fitting algorithms and so may be more rigid in fitting a diverse range of artifacts, but it may eliminate the artifact cleanly as well.

\section{Acknowledgements}

This research was made possible by the support of Dr. Aysegul Gunduz and the J. Crayton Pruitt Family Department of Biomedical Engineering at the University of Florida. 


\section{References}

Allen, D.P., Stegemöller, E.L., Zadikoff, C., Rosenow, J.M., MacKinnon, C.D. (2010). Suppression of deep brain stimulation artefacts from the electroencephalogram by frequency-domain Hampel filtering. Clinical Neurophysiology, 121 (8), 1227-1232.

Basir-Kazeruni, T., Vlaski S., Salami H., Sayed A.H., Markovi'c D. (2017). A Blind Adaptive Stimulation Artifact Rejection (ASAR) Engine for Closed-Loop Implantable Neuromodulation Systems. 8th International IEEE EMBS Conference on Neural Engineering. Shanghai, China, May 25 - $28,2017$.

Culaclii, S., Kim, B., Lo, Y., \& Liu, W. (2016). A hybrid hardware and software approach for cancelling stimulus artifacts during same-electrode neural stimulation and recording. 2016 38th Annual International Conference of the IEEE Engineering in Medicine and Biology Society (EMBC), 6190-6193.

Hashimoto, T., Elder, C.M., Vitek, J.L. (2002). A template subtraction method for stimulus artefact removal in high-frequency deep brain stimulation. Journal of Neuroscience Methods, 113, 181186.

Harding, G.W. (1991) A method for eliminating the stimulus artifact from digital recordings of the direct cortical response. Computational Biomedical Research, 24 (2),183-195.

Limnuson, K., Lu, H., Chiel, J. and Mohseni, P. (2014). Real-Time Stimulus Artifact Rejection Via Template Subtraction. IEEE Transactions on Biomedical Circuits and Systems 8(3), 391-400. doi: 10.1109/TBCAS.2013.2274574

Pearson, R.K. (2002). Outliers in process modeling and identification. IEEE Transactions on Control System Technologies, 2002, 55-63.

Peterson, E.J., Dinsmoor, D.A., Tyler, D.J., \& Denison, T.J. (2016). Stimulation artifact rejection in closed-loop, distributed neural interfaces. ESSCIRC Conference 2016: 42nd European Solid-State Circuits Conference, 233-236.

Sun, L. \& Hinrichs, H. (2016). Moving average template subtraction to remove stimulation artefacts in EEGs and LFPs recorded during deep brain stimulation. Journal of Neuroscience Methods, 266, 10.1016/j.jneumeth.2016.03.020.

Sun, L., Hinrichs, H. (2009). Simultaneously recorded EEG/fMRI: removal of gradient artefacts by subtraction of head movement related average artefact waveforms? Human Brain Mapping, 30 (10), 3361-3377.

Wagenaar, Daniel \& Potter, Steve. (2002). Real-time multi-channel stimulus artifact suppression by local curve fitting. Journal of Neuroscience Methods, 120, 113-20. 10.1016/S0165-0270(02)00149-8. 